University of Massachusetts Amherst ScholarWorks@UMass Amherst

2016

\title{
Direct-Breastfeeding in the Neonatal Intensive Care Unit and Breastfeeding Duration for Premature Infants
}

Carrie-Ellen Briere

University of Massachusetts Amherst, cbriere@umass.edu

Jacqueline M. McGrath

University of Connecticut

Xiaomei Cong

University of Connecticut

Elizabeth Brownell

University of Connecticut

Regina Cusson

University of Connecticut

Follow this and additional works at: https://scholarworks.umass.edu/nursing_faculty_pubs

Part of the Maternal, Child Health and Neonatal Nursing Commons

\section{Recommended Citation}

Briere, Carrie-Ellen; McGrath, Jacqueline M.; Cong, Xiaomei; Brownell, Elizabeth; and Cusson, Regina, "Direct-Breastfeeding in the Neonatal Intensive Care Unit and Breastfeeding Duration for Premature Infants" (2016). Applied Nursing Research. 224.

https://doi.org/10.1016/j.apnr.2016.04.004 


\title{
Direct-Breastfeeding in the Neonatal Intensive Care Unit and Breastfeeding Duration for Premature Infants
}

\begin{abstract}
Aim: To explore the relationship between direct-breastfeeding in the Neonatal Intensive Care Unit (NICU) and breastfeeding duration after discharge.

Background: Initiating and maintaining breastmilk feeding is an important goal that begins in the NICU. Little is known about direct-breastfeeding in the NICU and its relation to breastfeeding duration.
\end{abstract}

Methods: Chart review of 46 infants ( $<32$ weeks gestational age or $<1500$ grams) whose mothers provided breastmilk.

Results: One month after discharge, mothers still providing breastmilk were more likely to have provided $\geq 1$ direct-breastfeed per day in the NICU $(21.16$, CI: 3.13-143.25, p<0.01) and had prior breastfeeding experience (OR: 9.16, CI: 1.02-82.34, p<0.05). At 4 months, mothers still providing breastmilk were more likely to have provided $\geq 1$ direct-breastfeed per day in the NICU (OR: 12.80, CI: 1.39-118.32, p<0.05).

Conclusions: Direct-breastfeeding in the NICU may play an essential role in preparing mothers for breastfeeding after discharge, thus potentially impacting breastfeeding duration.

Keywords: Breastfeeding, Premature infant, Neonatal Intensive Care Unit, Discharge, Outcome 
DIRECT-BREASTFEEDING AND BREASTFEEDING DURATION

\section{Introduction}

Breastmilk is the gold standard for nutrition in infants. Premature infants benefit from a human milk diet with decreased morbidity and better long term neurodevelopmental outcomes when compared to those fed formula (1-6). Maintaining breastfeeding to meet recommended health standards is an important public health issue for both full-term and premature infants (7). Premature birth and subsequent NICU admission often are unexpected and may occur prior to families determining their infant feeding choice. The medical risks associated with premature birth increases the necessity for a human milk diet and information on the importance of breastmilk for the premature infant should be given to families with anticipated premature delivery and those with an infant in the $\operatorname{NICU}(2-5)$.

In premature infants, breastmilk feedings are often initiated by tube delivery (naso- or orogastric) and then progress to oral feeding once the infants are developmentally able to coordinate sucking, swallowing and breathing (8-10). Once oral feedings are initiated, infants may receive oral feedings through a variety of methods (two common methods are by bottlefeeding and breastfeeding), and exclusive breastmilk feeding is recommended for the first six months of life $(1,6)$. Direct-breastfeeding is when an infant suckles at the mother's breast with either a nutritive or non-nutritive outcome. The transition to oral direct-breastfeeding will vary for every infant and mothers with premature infants are at an increased risk of experiencing breastfeeding difficulties; in particular, they have a greater risk of not meeting breastfeeding goals decided upon before delivery(11-13).

Direct-breastfeeding in the Neonatal Intensive Care Unit (NICU) may play an important role in preparing the mother and infant for direct-breastfeeding after discharge. Successful direct- 
DIRECT-BREASTFEEDING AND BREASTFEEDING DURATION

breastfeeding after NICU discharge can be defined as the successful transition from a combination of direct-breastfeeding and feeding expressed breastmilk by bottle to full directbreastfeeding (or transition to the mother's goal ratio of direct-breast and bottle). Since researchers have found that mothers who provide more direct-breastfeeding have an increased likelihood of maintaining longer durations of breastmilk feeding during NICU hospitalization $(14,15)$, the act of direct-breastfeeding must be considered important in maintenance of long term breastfeeding duration.

The association of direct-breastfeeding in the NICU with breastfeeding outcomes is essential to explore so that NICU providers can understand the importance of supporting directbreastfeeding. Encouraging mothers to increase direct-breastfeeding opportunities while hospitalized is potentially a low cost, targeted intervention to increase breastfeeding success after discharge with the goal of reaching optimal recommendations for duration. Therefore, the purpose of this study was to explore the relationship between the frequency of directbreastfeeding in the NICU and breastfeeding duration after discharge.

\section{Methods}

\section{Study Design and Setting}

This study was conducted by retrospective chart review after approval from the Institutional Review Boards at the study hospital and university. The study location was a level IV urban NICU in New England with 32 patient beds. This study was the next phase in a sequence of studies that examined the characteristics and incidence of direct-breastfeeding in this NICU(16). Breastfeeding and other relevant data were routinely documented during NICU hospitalization, and follow-up data were obtained from follow-up lactation phone calls and 
DIRECT-BREASTFEEDING AND BREASTFEEDING DURATION

neurodevelopmental follow-up appointments at four and eight months corrected gestational age (CGA). The study site provides routine follow-up lactation phone support once per week until one month post-discharge home for every infant receiving any breastmilk.

\section{Sample Selection}

A convenience sample of eligible infants born between January 1, 2012 and December 31, 2012 was used in this retrospective review. Included infants were those eligible for neurodevelopmental follow-up (<32 weeks gestational age [GA] at birth, or <1500 grams). Infants were selected from the sample of infants that were eligible for the first phase of the study ( $<34$ weeks GA at birth, received maternal breastmilk for $\geq 7$ days, and discharged home with their mother on full oral feedings)(16). For this study, in addition to the requirement that infants meet criteria for follow-up, infants were also excluded if they had not continued to receive maternal breastmilk at the introduction of oral feeding (these infants could not receive any directbreastfeeding). The initial sample was 88 infants from the first study(16). After applying the additional inclusion/exclusion criteria for this study, 46 infants were eligible and included in the final analysis. See Figure 1.

\section{Measures.}

Direct-Breastfeeding in NICU: For the purposes of this study, direct-breastfeeding was when an infant suckled directly at the mother's breast. Quantity of milk transfer was not a variable of interest within this study. Oral feeding method is routinely documented by the nurse or lactation consultant for each feeding. Each feeding was only coded once and when an infant received a direct-breastfeeding followed by a bottle supplementation, the feeding was defined as a directbreastfeeding. 
DIRECT-BREASTFEEDING AND BREASTFEEDING DURATION

Frequency of Direct-Breastfeeding: All oral feedings were recorded from the first oral feeding until discharge. The number of direct-breastfeedings were divided by all oral feeding opportunities prior to discharge. To assist in understanding the clinical implications of breastfeeding practices, in the first study we divided infants into one of two groups to describe their direct-breastfeeding while in the NICU(16). Infants were divided at the median percentage amount of direct-breastfeeding which translated to the first group receiving < 1 direct-breastfeed per day in the NICU and the other group receiving $\geq 1$ direct-breastfeed per day (after oral feeding initiation). The median of this smaller sample was similar to that of the larger sample and the same clinical interpretation was used.

Breastfeeding Duration: The first breastfeeding follow-up call occurs two days after discharge, and then continues weekly until one month after discharge. Breastfeeding is recorded as "any breastfeeding" (exclusive and non-exclusive breastmilk feeding). Breastfeeding duration was also assessed by data collected from the NICU neurodevelopmental clinic appointments at 4 and 8 months CGA. At these appointments families report feeding type and method. Breastfeeding duration was categorized as breastmilk receipt; yes or no. Breastmilk receipt was categorized as "any" due to the lack of specific amounts available retrospectively in the charts.

\section{Data Collection Procedure}

Paper and electronic data were collected retrospectively through chart review by a NICU nurse (C.E.B.).These data were entered into an electronic database by a trained research assistant and $10 \%$ of entries were double coded to determine inter-rater agreement. Inter-rater agreement was $100 \%$ for the lactation follow-up data and $98 \%$ for the follow-up clinic data.

\section{Statistical Analysis}


DIRECT-BREASTFEEDING AND BREASTFEEDING DURATION

Statistical analyses were completed using SPSS Version 20. To eliminate redundancy of breastfeeding duration information in the first four lactation follow-up calls, the final call at one month after discharge was used, along with outcome at 4 and 8 months CGA. Bivariate analyses $\left(\chi^{2}\right)$ were conducted at each time point to examine the proportion of breastfeeding continuation (yes/no) with the direct-breastfeeding group ( $<1 /$ day or $\geq 1 /$ day) in the NICU as well as other potentially related factors from the literature and from the previous study(16). See Table 1. Variables with p-values $<0.05$ were chosen for inclusion into the multivariate backward selection Wald logistic regression models. Ethnicity and length of stay were forced into all multivariable models, regardless of the results of bivariate analyses.

A priori power analyses were completed to determine power for the available sample size. The sample size was able to achieve power of $87 \%$ (two sided; $\alpha=0.05$ ) with a difference in group proportion (of direct-breastfeeding group) of continued breastfeeding in Chi-squared analyses of 40 percent.

\section{Results}

\section{Sample Description}

The mean GA at birth was $296 / 7 \pm 2.5$ weeks (Range 25 3/7 - 33 3/7 weeks) with a mean birth weight of 1,280 \pm 358 grams (Range $706-2470$ grams). The mean length of stay was $58 \pm$ 27.5 days (Range 19-131 days). The sample included primarily white, non-Hispanic, first-time mothers. Table 1 presents additional details on sample characteristics of infants and mothers.

At discharge, $48 \%(n=22)$ of infants received an exclusively breastmilk diet (inclusive of infants with fortified calories), $17 \%(\mathrm{n}=8)$ received a mixed breastmilk and formula diet, and 


\section{DIRECT-BREASTFEEDING AND BREASTFEEDING DURATION}

$35 \%(\mathrm{n}=16)$ were discharged solely on formula. Of the 30 infants discharged home receiving some breastmilk, $90 \%(\mathrm{n}=27)$ had at least one follow-up phone contact with a lactation consultant. Breastmilk receipt declined during the month after discharge; of the 27 mothers contacted, $100 \%$ continued to provide breastmilk at the first call, $96 \%$ at the second, $95.7 \%$ at the third, and $84 \%$ at one month after discharge.

For Neonatal Follow-up, $83 \%(n=38)$ of the sample attended at least one NICU follow-up visit. More specifically, $78 \%(n=31)$ attended the four month visit and 63\% (n=29) attended the eight month visit. Thirty-eight percent $(n=9)$ of infants who were discharged home on breastmilk and returned for follow-up at 4 months CGA continued to receive some breastmilk and at 8 months CGA, $32 \%$ continued to receive breastmilk $(n=7)$.

\section{Direct-breastfeeding in the NICU and breastfeeding duration after discharge}

After discharge $51 \%(n=21 / 41)$ of infants continued to receive breastmilk at one month post-discharge, $26 \%(n=9 / 35)$ at 4 months CGA, and $23 \%(n=7 / 30)$ at 8 months CGA.

At one month after discharge, only two variables made a statistically significant contribution to the model (amount of direct-breastfeeding in the NICU and mothers with breastfeeding experience). Mothers who provided $\geq 1$ direct-breastfeeding per day in the NICU (OR: 21.16, CI: 3.13-143.25, p<0.01) or had prior breastfeeding experience (OR: 9.16, CI: 1.02$82.34, \mathrm{p}<0.05)$ were significantly more likely to continue to provide breastmilk at one month after discharge. See Table 2.

At 4 months CGA, only one variable made a statistically significant contribution to the model. Mothers who provided $\geq 1$ direct-breastfeeding per day in the NICU were significantly 
more likely to provide breastmilk at 4 months CGA (OR: 12.80, CI: 1.39-118.32, p<0.05). See Table 2.

At 8 months CGA no variables showed a significant impact with breastmilk receipt in bivariate analyses, but we forced direct-breastfeeding group, ethnicity, maternal age, and length of stay into the model; none independently predicted breastmilk receipt at 8 months CGA.

\section{Discussion}

Direct-breastfeeding in the NICU allows mothers to navigate breastfeeding a premature infant with the ability to receive support and education from trained staff. Conceptually, mothers who direct-breastfeed more in the NICU, will be more likely to direct-breastfeed at home after discharge. Previously we had not known of any researchers that examined the association of direct-breastfeeding in the NICU with breastfeeding duration after discharge. Results from this study support the importance of direct-breastfeeding in the NICU with long-term breastfeeding outcomes since mothers who provided $\geq 1$ direct-breastfeeding per day in the NICU were significantly more likely to have continued breastfeeding at one month after discharge and at 4 months CGA. These findings along with the results from other studies that examined breastfeeding and breastmilk receipt at discharge(14-16) support the importance of promoting direct-breastfeeding within the NICU. The clinical significance of the findings from this study is that if we can support mothers to directly-breastfeed at least once per day, these mothers and infants may have better long term breastfeeding outcomes. Supporting direct-breastfeeding once daily may seem insignificant to the practicing provider, however these results show that even this small intervention could possibly affect the breastfeeding duration for mother-infant dyads. 


\section{DIRECT-BREASTFEEDING AND BREASTFEEDING DURATION}

Smith and colleagues (15) found that site of care influences the amount of directbreastfeeding that occurs within the NICU and this may be related to cultural differences within the staff on breastfeeding knowledge and support. Staff education to improve consistency of breastfeeding advice and counseling is essential because mothers have reported contradicting advice from different nurses in the NICU (17). Pineda found that staff education can impact breastfeeding outcomes because after breastfeeding education for staff, the amount of directbreastfeeding increased in their unit (18). Evidence-based knowledge on the importance of direct-breastfeeding and appropriate interventions to support direct-breastfeeding are needed for NICUs. Interestingly, Smith and her colleagues also reported that mothers in their study recognized that their infant was discharged early at the expense of breastfeeding because NICU staff encouraged bottle-feeding over breastfeeding under the misconception that bottling leads to sooner discharge (17). These mothers thought that breastfeeding would be easier at home and were left to manage breastfeeding issues after discharge without the support of NICU staff (17). There currently is not enough evidence to support the idea that bottle-feeding leads to sooner discharge.(19) Additionally, mothers have reported that breastmilk and breastfeeding were often disconnected concepts in the NICU with a large emphasis on the benefit of breastmilk but minimal support and discussion on the importance of direct-breastfeeding (17). The importance of direct-breastfeeding in the NICU with breastfeeding outcomes after discharge is evident from this study and interventions to increase direct-breastfeeding in conjunction with the provision of breastmilk in the NICU are needed.

\section{Future Research}

Initial findings from this study suggest the importance of direct-breastfeeding in the NICU on breastfeeding duration. Future research should include prospective trials with an 
DIRECT-BREASTFEEDING AND BREASTFEEDING DURATION

intervention targeting increased direct-breastfeeding in the NICU. A randomized, controlled trial could provide additional details on educational interventions developed to increase directbreastfeeding in the NICU for both staff and for mothers and families. Larger sample sizes with more specific breastfeeding measures should also be considered. In addition, a mixed methods approach would provide important parent and staff perspectives on barriers and facilitators to direct-breastfeeding in the NICU. These perspectives could help shape future interventions to increase and support direct-breastfeeding.

\section{Limitations}

This retrospective study provided results from one NICU with a limited sample size of convenience (retrospective). Attrition due to follow-up should be expected in any study with follow-up and the sample size decreased at each time point. Due to this, the findings from the 8 month follow-up need further future evaluation. Future studies need greater sample sizes due to expected attrition. Although the data are from one institution, these findings are more focused on the association between direct-breastfeeding in the NICU and breastfeeding duration, and these can be examined in the context of other institutions as well. Since this study was retrospective, we were unable to account for potential unmeasured confounding variables.

This study included a high proportion (41\%) of infants from multiple births. Premature multiples receive lower amounts of breastmilk and breastfeeding when compared to full-term singletons and multiples, as well as premature singletons (20) and the high proportion of multiples in this sample may underrepresent the breastfeeding duration of the premature singleton (other samples with lower amounts of multiple births may find higher rates of breastmilk continuation). 
DIRECT-BREASTFEEDING AND BREASTFEEDING DURATION

\section{Conclusion}

Supporting mothers to provide at least one direct-breastfeeding per day is an intervention that NICU staff can help mothers to achieve. NICU staff need to know that although the receipt of breastmilk is important regardless of delivery method, direct-breastfeeding has an important role with additional benefits over feeding breastmilk by bottle (21). Importantly, directbreastfeeding in the NICU may play an essential role in preparing mothers for breastfeeding after discharge and thus impacting duration of breastfeeding. Although the premature infant can often not begin oral feeding at birth, the successful initiation of direct-breastfeeding is an important supportive intervention that NICU caregivers can facilitate.

\section{Acknowledgements}

We wish to thank Sharon Casavant for her assistance in data collection. This work was supported by a grant from the Mu chapter of Sigma Theta Tau International (to cover expenses involved with data collection and dissemination).

\section{Funding Details}

The study sponsor had no involvement in study design, collection (other than covering costs), analysis or interpretation of data; in the writing of the manuscript; or in the decision to submit the manuscript for publication. 


\section{DIRECT-BREASTFEEDING AND BREASTFEEDING DURATION}

\section{References}

1. American Academy of Pediatrics. Breastfeeding and the use of human milk. Pediatrics. 2012;129(3):e827-e41.

2. Sisk P, Lovelady C, Dillard R, Gruber K, O'shea T. Early human milk feeding is associated with a lower risk of necrotizing enterocolitis in very low birth weight infants. Journal Of Perinatology. 2007;27(7):428-33.

3. Okamoto T, Shirai M, Kokubo M, Takahashi S, Kajino M, Takase M, et al. Human milk reduces the risk of retinal detachment in extremely low-birthweight infants. Pediatrics international : official journal of the Japan Pediatric Society. 2007;49(6):894-7.

4. Vohr B, Poindexter B, Dusick A, McKinley L, Higgins R, Langer J, et al. Persistent beneficial effects of breast milk ingested in the neonatal intensive care unit on outcomes of extremely low birth weight infants at 30 months of age. Pediatrics. 2007;120(4):e953-9.

5. Vohr B, Poindexter B, Dusick A, McKinley L, Wright L, Langer J, et al. Beneficial effects of breast milk in the neonatal intensive care unit on the developmental outcome of extremely low birth weight infants at 18 months of age. Pediatrics. 2006;118(1):e115-23.

6. Academy of Breastfeeding Medicine Board of Directors. Position on breastfeeding. Breastfeeding medicine : the official journal of the Academy of Breastfeeding Medicine. 2008;3(4):26770.

7. United States Department of Health and Human Services. Infant Care Objectives Washington, DC2014. Available from: http://www.healthypeople.gov/2020/topicsobjectives2020/objectiveslist.aspx?topicld=26.

8. National Association of Neonatal Nurses. Infant directed oral feeding for premature and critically ill hospitalized infants, guideline for practice. Chicago, IL: 2013.

9. Briere C-E, McGrath J, Cong X, Cusson R. State of the Science... A contemporary review of feeding readiness in the preterm infant. Journal of Perinatal \& Neonatal Nursing. 2014;28(1):51-8. 10. Jones LR. Oral feeding readiness in the neonatal intensive care unit. Neonatal Network. 2012;31(3):148-55.

11. Callen J, Pinelli J. A review of the literature examining the benefits and challenges, incidence and duration, and barriers to breastfeeding in preterm infants. Advances in Neonatal Care. 2005;5(2):72-88.

12. Dougherty $\mathrm{D}$, Luther $\mathrm{M}$. Birth to breast -- a feeding care map for the NICU: helping the extremely low birth weight infant navigate the course. Neonatal Network. 2008;27(6):371-7.

13. Cahill JB, Wagner CL. Challenges in breastfeeding, maternal considerations. Contemporary Pediatrics. 2002;19(5):94-110.

14. Pineda RG. Direct breast-feeding in the neonatal intensive care unit: is it important? Journal of Perinatology. 2011;31(8):540-5.

15. Smith M, Durkin M, Hinton V, Bellinger D, Kuhn L. Initiation of breastfeeding among mothers of very low birth weight infants. Pediatrics. 2003;111(6):1337-42.

16. Briere CE, McGrath JM, Cong X, Brownell E, Cusson R. Direct-Breastfeeding Premature Infants in the Neonatal Intensive Care Unit. Journal of human lactation : official journal of International Lactation Consultant Association. 2015;31(3):386-92.

17. Niela-Vilén $\mathrm{H}$, Axelin A, Melender H, Salanterä S. Aiming to be a breastfeeding mother in a neonatal intensive care unit and at home: a thematic analysis of peer-support group discussion in social media. Maternal \& Child Nutrition. 2014.

18. Pineda RG, Foss J, Richards L, Pane CA. Breastfeeding changes for VLBW infants in the NICU following staff education. Neonatal Network. 2009;28(5):311-9. 
19. Briere CE. Breastfed or bottle-fed: who goes home sooner? Advances in neonatal care : official journal of the National Association of Neonatal Nurses. 2015;15(1):65-9.

20. Geraghty SR, Pinney SM, Sethuraman G, Roy-Chaudhury A, Kalkwarf HJ. Breast milk feeding rates of mothers of multiples compared to mothers of singletons. . Ambulatory Pediatrics. 2004;4(3): 226-31.

21. Buckley KM, Charles GE. Benefits and challenges of transitioning preterm infants to at-breast feedings. International Breastfeeding Journal. 2006;1(13). 